\title{
Spatial Heterodyne Spectro-Polarimetry Systems for Imaging Key Plasma Parameters in Fusion Devices
}

\author{
John HOWARD, Ahmed DIALLO, Roger JASPERS ${ }^{1)}$ and Jinil CHUNG $^{2)}$ \\ Plasma Research Laboratory, Australian National University. Canberra ACT 0200, Australia \\ 1) FOM institute for Plasmaphysics Rijnhuizen, 3430 BE Nieuwegein, The Netherlands \\ ${ }^{2)}$ National Fusion Research Institute, Gwahangno 113, Yusung-Gu, Daejeon, 305-333, Korea
}

(Received 19 January 2009 / Accepted 8 July 2009)

\begin{abstract}
Imaging diagnostics systems are very important to aid the understanding of core and edge confinement in 3D helical magnetic devices. In this paper we consider recent developments in optical "coherence imaging" interferometric systems that open new diagnostic capabilities for next generation devices, with particular focus on Motional Stark Effect (MSE) imaging. We present preliminary results obtained using a hybrid spatio-temporal heterodyne snapshot imaging polarimeter-interferometer for motional Stark effect imaging of the $q$-profile in the TEXTOR tokamak.

(C) 2010 The Japan Society of Plasma Science and Nuclear Fusion Research
\end{abstract}

Keywords: polarimetry, spectroscopy, Motional Stark Effect, coherence, interferometry

DOI: $10.1585 /$ pfr.5.S1010

\section{Introduction}

Motional Stark Effect spectro-polarimetry has become a routine diagnostic for high power fusion devices that employ diagnostic or heating neutral beams. In tokamaks, measurement of the polarization state of the Stark split multiplet is used to infer the internal toroidal current density profile $[1,2]$, while for stellarators MSE can be used to help determine the plasma equilibrium. Until now, because of various technical limitations, especially in low field compact systems where the Stark multiplet is difficult to resolve or is contaminated by other spectral features, MSE systems have been limited to 10 or 20 discrete channels viewing positions across the injected beam.

The MSE technique relies on the splitting of the Doppler-shifted neutral beam Balmer $\alpha$ light into orthogonally polarized $\sigma$ and $\pi$ components as a result of the motion-induced strong electric field $\boldsymbol{E}=\boldsymbol{v} \times \boldsymbol{B}$ experienced in the rest frame of the neutral atoms. The Doppler shift, which arises due to observation at an angle to the energetic beam, conveniently shifts the multiplet away from the background $\mathrm{H}_{\alpha}$ radiation. When viewed in a direction perpendicular to $\boldsymbol{E}$ the Stark split $\sigma$ and $\pi$ components are polarized respectively perpendicular and parallel to the direction of $\boldsymbol{E}$. When viewed along $\boldsymbol{E}$ the $\sigma$ components are unpolarized and the $\pi$ components have no brightness. The Stark separation of adjacent Balmer alpha spectral components varies as $\Delta \lambda_{S}=2.7574 \times 10^{-8} E$ nm where $E=|\boldsymbol{v} \times \boldsymbol{B}|$ is the induced electric field [3]. Integrated over wavelength, the Stark multiplet is nett unpolarized and no orientational information can be obtained.

The magnetic field pitch angle is usually estimated by

author'se-mail: john.howard@anu.edu.au isolating and measuring the polarization direction of the central cluster of $\sigma$ lines. This requires a tunable narrowband filter to spectrally resolve the multiplet in order to obtain a nett polarization that is analysed by a modulated polarimeter. Every spatial channel thus requires a dedicated filter whose passband must be optimized by tilt or thermal tuning. Recently we have proposed a variety of optical systems that can capture the full polarimetric information about the entire Stark multiplet in a single snapshot [4]. This is achieved by using interfero-polarimetric methods to produce orthogonal spatial carrier fringes which encode the optical coherence (spectrum) and polarimetric information.

In the slightly simpler scheme described here, a spatial heterodyne polarization interferometer is used to provide spectral discrimination and to imprint interference fringes on an image of the neutral beam. The contrast and phase of the fringes depend on the spectral separation of the Stark components and their mean wavelength. A front end polarimeter which employs a switching liquid crystal waveplate modulates the fringe phase in proportion to the local polarization orientation of the entire Stark multiplet. Successive independent images can then be phase demodulated to recover an image of the local magnetic field pitch angle. Because of the spatial encoding and the ability to accept a relatively wide spectral window, it becomes feasible to undertake two-dimensional magnetic field imaging. Moreover, the resulting extracted image of the Stark multiplet polarization orientation is insensitive to arbitrary unpolarized spectral contamination such as the wing of the $\mathrm{H}_{\alpha}$ emission, or leakage from adjacent beam energy components. Under certain conditions, it is also insensitive to background radiation that has become polarized due to re- 
flections from various surfaces.

This paper is organized as follows. Section 2 gives a brief description of the optical system, its operating principle and its implementation on TEXTOR for observations of the $\mathrm{H}_{\alpha}$ multiplet. First observations are presented in Sec. 3. The experimental images when compared with modeling results, suggest that line-of-sight integration effects may be important for the interpretation of the pitch angle images. The results also suggest a number of tests and crosschecks which will be undertaken during a dedicated day of operations in March 2009. These issues are considered in Sec. 4.

\section{MSE Imaging Spectro-Polarimeter}

\subsection{Measurement principle}

Figure 1 (a) shows the optical arrangement for a simple polarization interferometer comprising a polarizer, birefringent delay plate (of phase delay $\phi$ ) with fast axis at $45^{\circ}$ to the polarizer axis, and final analyzer parallel to the first polarizer. Within a factor, the interferometric signal is

$$
S=I_{0}(1+\zeta \cos \phi),
$$

where $I_{0}$ is the brightness, $\zeta$ is the fringe visibility at optical delay $\phi=2 \pi L B / \lambda_{0}, L$ is the birefringent plate thickness, $B$ is the birefringence and $\lambda_{0}$ is the wavelength.

If the light source is already polarized, the first polarizer can be removed and the signal becomes

$$
S=I_{0}(1+\zeta \cos 2 \theta \cos \phi),
$$

where $\theta$ is the polarization angle with respect to the final analyzer axis. If the wave-plate is replaced with a
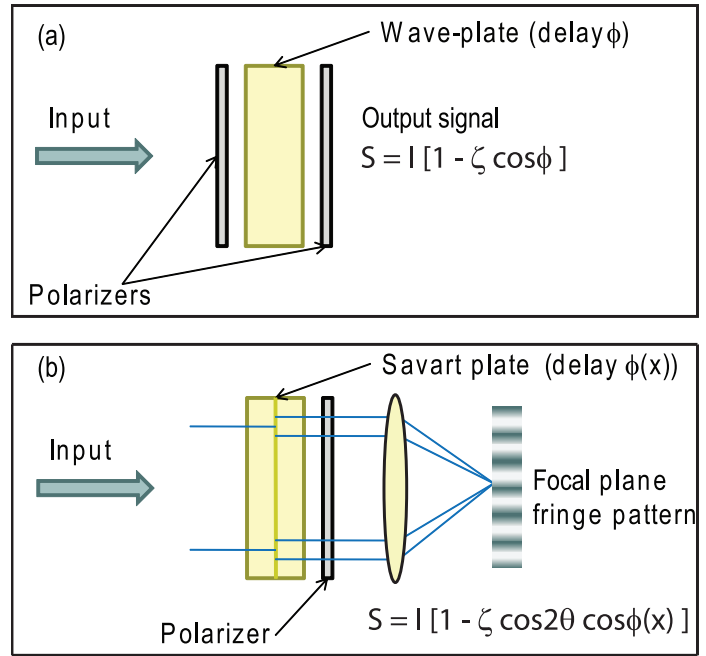

Fig. 1 (a) A simple polarization interferometer. The fringe visibility $\zeta$ depends on the optical coherence at phase delay $\phi$. (b) When the input light is already polarized, the first polarizer can be omitted. The fringe contrast then also depends on the input polarization orientation with respect to the final analyzer. A shearing Savart wave-plate produces a sinusoidal fringe pattern in the lens focal plane.
Savart shearing plate [5], the phase delay becomes a function of position and an interference fringe pattern is formed in the focal plane of a final imaging lens. The fringes carry the polarization orientation through the amplitude term $\zeta \cos 2 \theta$.

With the installation of a quarter wave plate with fast axis parallel to the analyzer as the first element in the optical chain, the polarization orientation is shifted to the phase domain and the detected image is

$$
S=I_{0}[1+\zeta \cos (\phi-2 \theta)]
$$

Because the phase delay $\phi(x)$ is generally not known absolutely, it is necessary to introduce a modulating element in order to extract the polarization orientation. In our case, we insert a ferroelectric liquid crystal (FLC) half waveplate between the first quarter wave plate and the following optical delay plate (see Fig. 2). Nominally the FLC fast axis is aligned parallel to the delay plate and $\phi$ becomes $\phi+\pi$. By reversing the polarity of a low voltage bias of order $5 \mathrm{~V}$ across the FLC cell, the birefringent axes rotate through $45^{\circ}$ so that the fast axis is now crossed with the axes of the quarter wave-plate, reversing the sense of the quarter-wave delay and changing the sign of the term $2 \theta$ in Eq. (3). Though FLC switches can operate at frequencies up to $20 \mathrm{kHz}$ or more, the limit on temporal resolution in our case is set by the requirement that the FLC be synchronized to the camera frame rate of $\sim 50 \mathrm{~Hz}$. The images in the two FLC states are respectively

$$
\begin{aligned}
& S_{1}=I_{0}[1-\zeta \cos (\phi-2 \theta)] \\
& S_{2}=I_{0}[1+\zeta \cos (\phi+2 \theta)]
\end{aligned}
$$

and it is clear that a suitable demodulation procedure applied to successive images will produce an image of $4 \theta$.

Because the MSE multiplet is nett unpolarized the signs of the $\pi$ and $\sigma$ contributions to the image of the complete multiplet are opposite and, in the absence of spectral discrimination, the fringe pattern vanishes. However, by

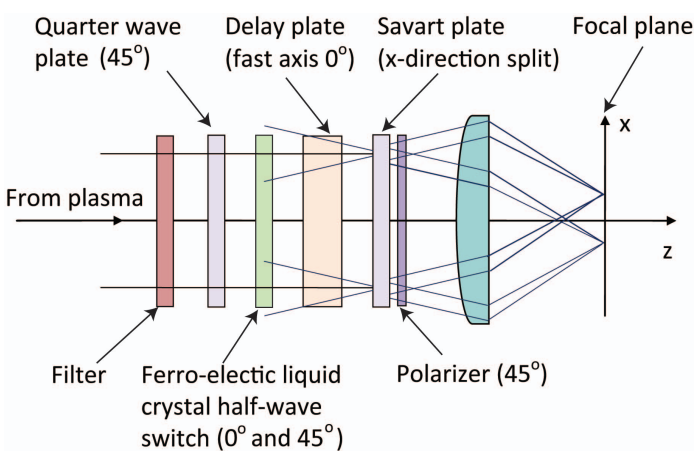

Fig. 2 Layout of the hybrid spectro-polarimeter. The quarterwave plate and FLC switch constitute the polarimeter. The primary delay plate and polarizer provide spectral discrimination. The Savart plate imprints a sinusoidal spatial carrier wave in the $x$-direction. 
appropriately choosing the optical delay offset $\phi$ it is possible to maximize the difference between $\zeta_{\pi}$ and $\zeta_{\sigma}$ to ensure good nett fringe visibility, even when the ratio of the separation to width of the multiplet components is small [4].

\subsection{The TEXTOR optical system}

The TEXTOR neutral beam and viewing geometry is depicted in Fig. 3. A custom turning prism mounted in vacuum is used to direct light from the beam through a fusedsilica window into the spectro-polarimeter. The instrument is constructed from optical polarizing components of diameter $25 \mathrm{~mm}$ housed in a $40 \mathrm{~mm}$ diameter turret that screws into the filter thread of a $17 \mathrm{~mm}$ focal length wide-angle Cmount imaging lens (see Fig. 6). The lens (focused at infinity) forms an image of the plasma onto an $8 \mathrm{~mm} \times 6 \mathrm{~mm}$ imaging fibre bundle array which transports the image to a Cooke sensicam CCD camera. The turret, lens and fibre cable are inserted into a re-entrant port and positioned adjacent to the fused-silica vacuum window.

At the fiber cable exit a $55 \mathrm{~mm}$ focal length F-mount lens collimates the light through tiltable $2 \mathrm{~nm}$ pass-band interference filter centered on $662.5 \mathrm{~nm}$. The filter is adjusted so that the centre wavelength approximately tracks the variation in Doppler shift of the multiplet across the beam image. A final $50 \mathrm{~mm}$ focal length F-mount lens

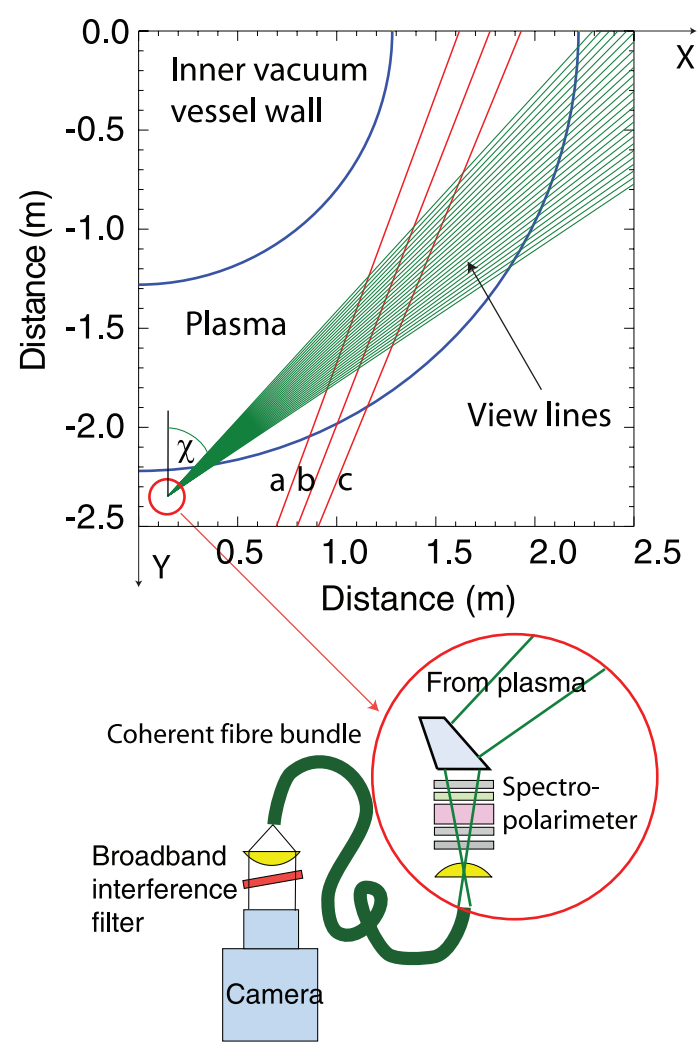

Fig. 3 The TEXTOR system geometry. The neutral beam spans the region designated by the lines labeled ' $a$ ', 'b' and 'c'. A coherent fibre bundle transports the image to a broadband interference filter and CCD camera synchronized to the FLC switch. forms the final image.

We have constructed a numerical model of the TEXTOR system to calculate the expected spectrum and associated nett fringe contrast versus optical delay as a function of viewing angle to the beam [4]. The results shown in Fig. 4 indicate that an optical delay of approximately 1000 waves at $662 \mathrm{~nm}$ should give adequate nett fringe contrast across the viewing region. The higher contrast at the plasma edge is due to the wider separation of the multiplet components in that region. The delay is obtained using a field-widened lithium niobate delay plate of total thickness $6 \mathrm{~mm}$. A conventional calcite Savart shearing plate of thickness $1 \mathrm{~mm}$ is used to generate the spatial heterodyne fringe pattern in the focal plane of the focusing lens.

Figure 5 (a) shows the interior of the TEXTOR vacuum vessel as viewed by the spectro-polarimeter. Various image features such as port openings and flanges are used to calibrate the observed field of view. The $2 \times 2$ binned images $(688 \times 520$ pixels $)$ have been cropped to show only regions accepted by the spectro-polarimeter optical system. Figures 5 (b) and (c) show images of plasma light in the $662 \mathrm{~nm}$ filter pass-band without and in the presence of the neutral heating beam respectively. The color scales are in units of photo-electrons and the image exposure time was $4 \mathrm{~ms}$. Note that the plasma light image shows no evi-
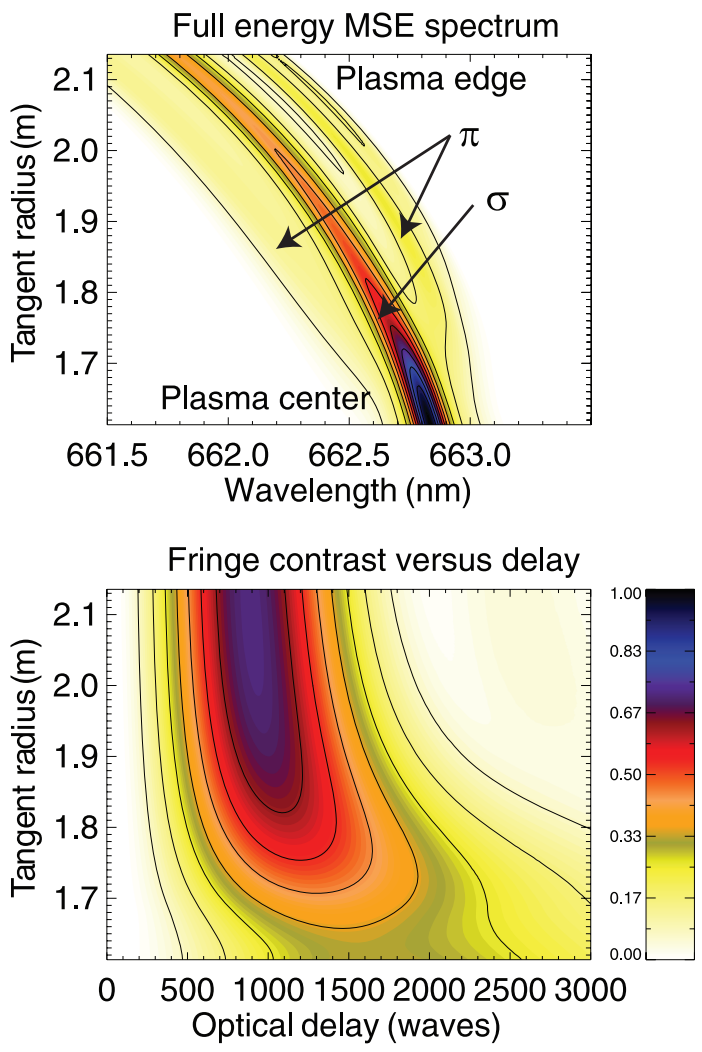

Fig. 4 Top: The model MSE spectrum for the imaging arrangement on TEXTOR and Bottom: the corresponding fringe contrast versus optical delay. The fringe contrast is poorer near plasma center where the separation between Stark components is small. 

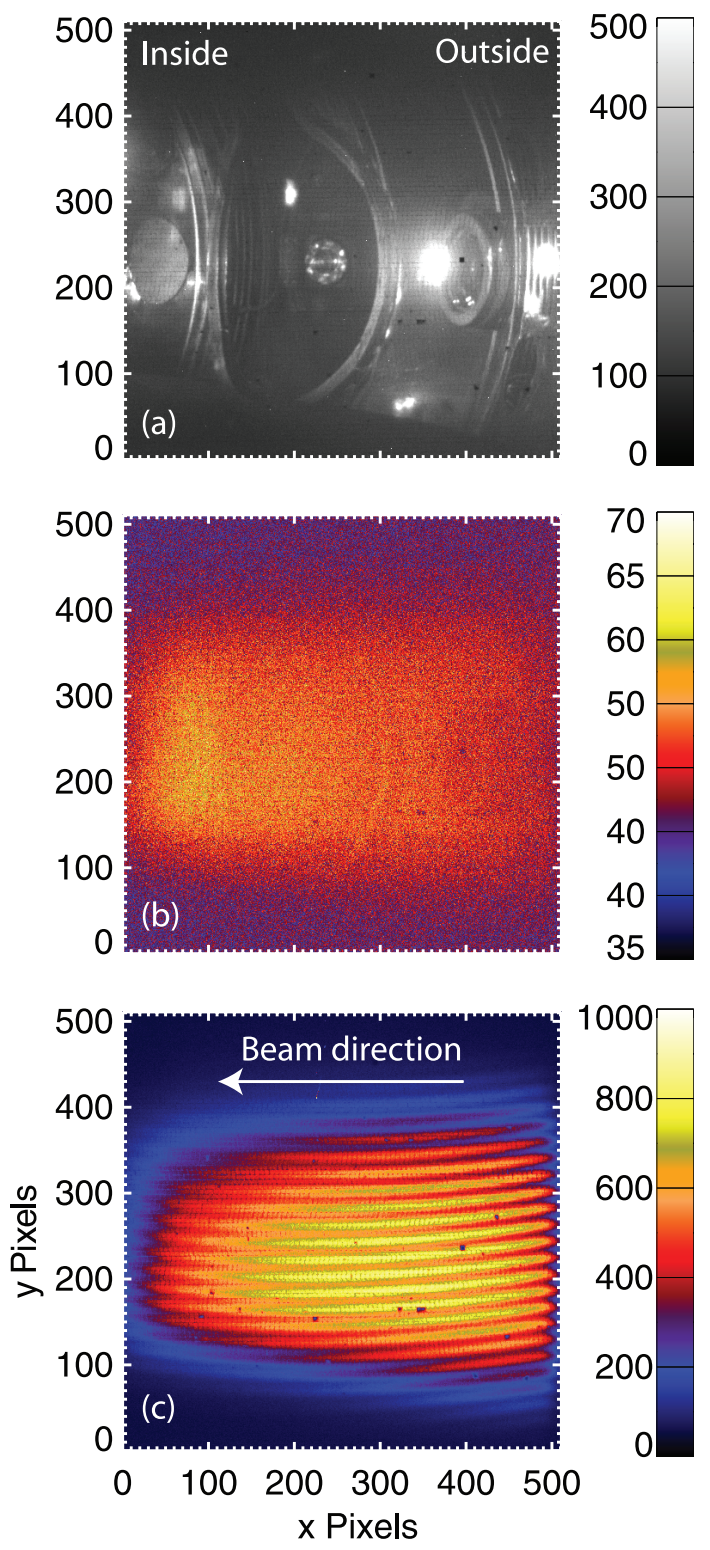

Fig. 5 (a) Image of interior of TEXTOR vacuum vessel as recorded by the spectro-polarimeter. (b) Plasma emission within the $660 \mathrm{~nm}$ filter pass-band (no neutral beam emission) for discharge \#108248. Notice the absence of interference fringes. (c) Image of the neutral beam. Optical fiber cable imperfections are visible. Visible interference fringes indicate that the light is polarized. Successive images can be demodulated for the polarization orientation.

dence of interference fringes, indicating the absence of polarized features of spectral width comparable to the MSE multiplet components. As evident in Fig. 5 (c), the spatial fringes have been oriented parallel to the horizontal midplane in order to maximize the radial spatial resolution. The resulting radial resolution $\sim 3 \mathrm{~mm}$ is set by fibre cable resolution and image binning, while the vertical resolution of $\sim 15-20 \mathrm{~mm}$ is set by fringe period. While the observed fringe curvature can be eliminated by field-widening the Savart plate [5], it is of no consequence in the present optical configuration where the fringe phase can be recovered by demodulating the images column-by-column.

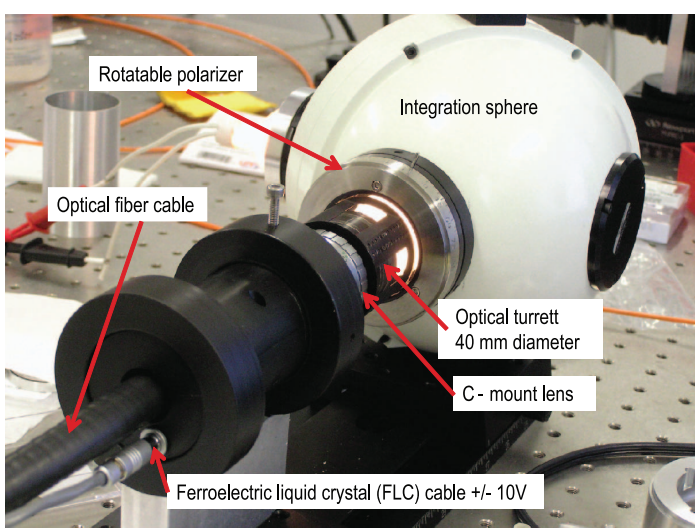

Fig. 6 A photograph of the optical head during calibration procedure. The optical head is inserted into a re-entrant port that views the plasma and neutral beam through a turning prism and fused silica window.

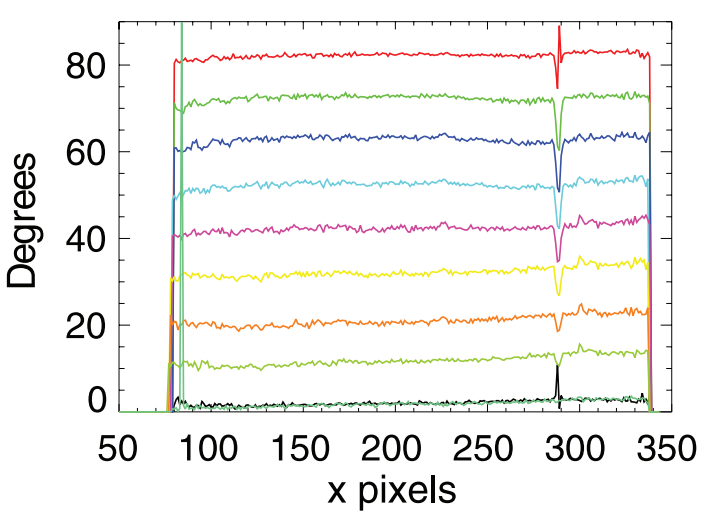

Fig. 7 Central horizontal slices for a sequence of calibration phase images for polarizer angles between $0^{\circ}$ and $90^{\circ}$ in increments of $10^{\circ} \pm 1^{\circ}$. The image discontinuities are due to a faulty optical cable pixel.

\subsection{Optical system calibration}

The system polarimetric response is calibrated by filling the field-of-view using Balmer-alpha light from a hydrogen lamp and replacing the $662 \mathrm{~nm}$ filter with a filter at $656 \mathrm{~nm}$. A rotatable polarizer is used to vary the incident polarization and image sequences are acquired and processed for a range of polarizer angles spanning $90^{\circ}$. A typical calibration arrangement is shown in Fig. 6. Central horizontal slices for a sequence of calibration phase images for polarizer angles between $0^{\circ}$ and $90^{\circ}$ in increments of $10^{\circ} \pm 1^{\circ}$ are shown in Fig. 7. We have implemented both wavelet and Hilbert transform demodulation algorithms. The latter method, which is based on fast Fourier transforms and is therefore quite fast, generally suffices when the signal to noise ratio is good (as is the case for data presented in this paper). Image lines show typical root mean square phase noise of $\sim 0.5^{\circ}$. Small systematic departures from uniformity may be due to component alignment inaccuracies which will be addressed in the next version of the 
instrument. These distortions, which are nevertheless quite small, have not been compensated in the results presented in this article.

\section{Results}

To illustrate performance the system performance we consider an image sequence for discharge \#108250 during which the toroidal magnetic field was $2.25 \mathrm{~T}$ on axis and the toroidal current was $\sim 350 \mathrm{kA}$. For this discharge, the camera exposure time was $2 \mathrm{~ms}$, the frame period was $\sim 50 \mathrm{~ms}$ and the image size was $344 \times 260$ pixels. The time history of the interferogram brightness averaged over a central cell of dimensions $10 \times 10$ pixels is shown in Fig. 8 . Note the strong modulation in the beam brightness and accompanying phase modulation of amplitude $\sim 0.2$ radians corresponding to a periodic modulation in the beam energy of amplitude $\sim 0.4 \mathrm{keV}$-consistent with independent beam energy measurements. It is important that the image exposure time be less than the modulation period in order to maintain good fringe visibility.

The interferograms have been demodulated using a wavelet-based algorithm and images of the fringe visibility and inferred polarization orientation $\theta$ versus image coordinate angles $(\chi, \psi)$ where $\chi$ is the angle between the line of sight and tokamak $Y$-axis as shown in Fig. 3 and $\psi$ is the vertical angle above the horizontal midplane. Representative images taken at time $1.8 \mathrm{~s}$ in the discharge are shown in Fig. 9. As expected, the fringe contrast deteriorates towards the center of the plasma where the multiplet splitting decreases (compare with Fig. 4). We have not corrected for weak instrumental fringe contrast variations.

To interpret the experimental polarization tilt angle image, we have developed a numerical model to calculate the expected inclination distribution for the TEXTOR viewing geometry. We assume a toroidal current distribution of the form $j(r)=j_{0}\left(1-r^{2} / a^{2}\right)^{\gamma}$ and in the simulations reported here, have taken the value $\gamma=2$ for the exponent and the plasma minor radius is $a=0.47 \mathrm{~m}$. To calculate the polarization orientation, for each point in the field of view,

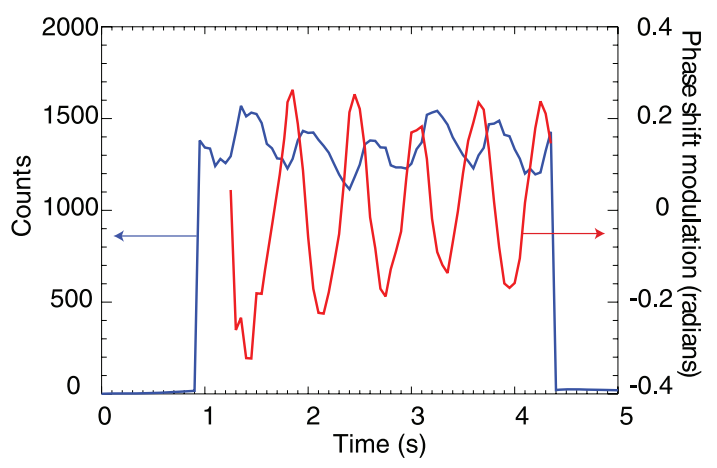

Fig. 8 Temporal behaviour of central image brightness and carrier phase. A strong modulation in the beam parameters is evident. See text for discussion. we project the local $\boldsymbol{E}=\boldsymbol{v} \times \boldsymbol{B}$ onto a measurement Cartesian coordinate system having $z$ axis connecting the measurement pixel and the field point and $x$-axis in the tokamak horizontal plane. Because the beam is extended both vertically and toroidally, the measured polarization angle at a given image point can be derived from the summation of the Stokes vectors describing the polarization state for all points on the $z$-axis that intersect the beam.

While the brightness weighting along the view line is unknown, it is nevertheless instructive to calculate the electric field orientation on vertical planes intersecting the beam on the beam axis (label ' $b$ ' in Fig. 3) and on planes labeled ' $a$ ' and 'c' in Fig. 3 that are displaced by $1^{\circ}$ either side of the axis ' $b$ ' relative to the model beam origin. These planes are at angles within the estimated divergence of the $50 \mathrm{keV}$ hydrogen heating neutral beam. The calculated polarization angle distributions are shown in Fig. 10. As for Fig. 9 (a), the color contours span the range $\left[80^{\circ}, 105^{\circ}\right]$ while, in each case, the vertical angular extent is set by the beam model and geometric perspective. We observe a strong positional dependence that suggests that path integration effects may be important in the TEXTOR case. Note that the three computed images taken together tend to capture many of the features observed in the experimental image and match satisfactorily the observed range of inclination angles.

It should be noted that the small systematic phase distortions evident in Fig. 7 have not been compensated in the phase image of Fig. 9. Moreover, inspection of the internal reflecting prism following the completion of this cam-
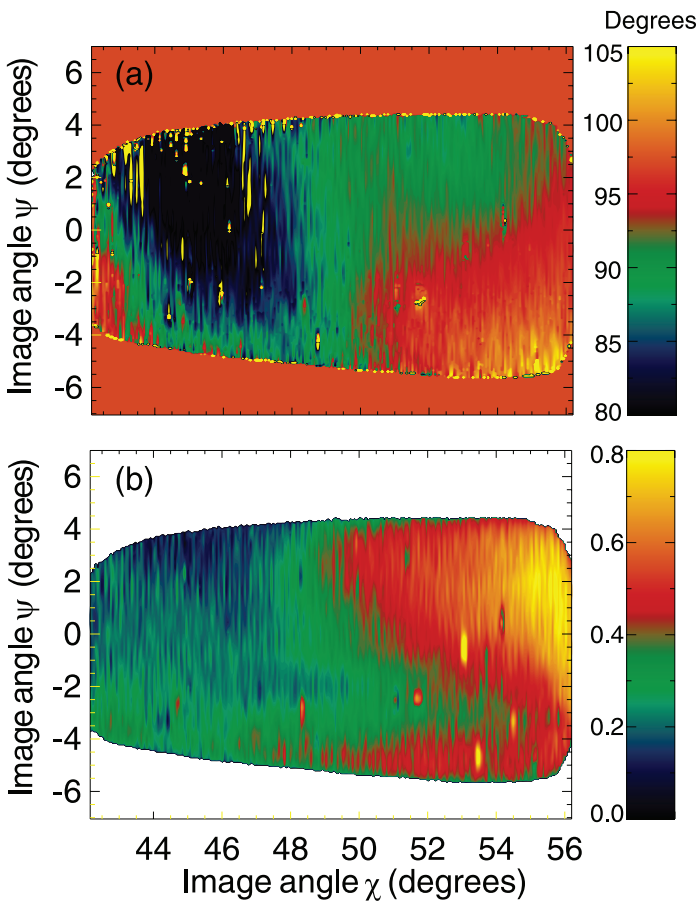

Fig. 9 (a) Demodulated polarization tilt angle image at $1.8 \mathrm{~s}$ and (b) corresponding fringe visibility image. See text for discussion. 

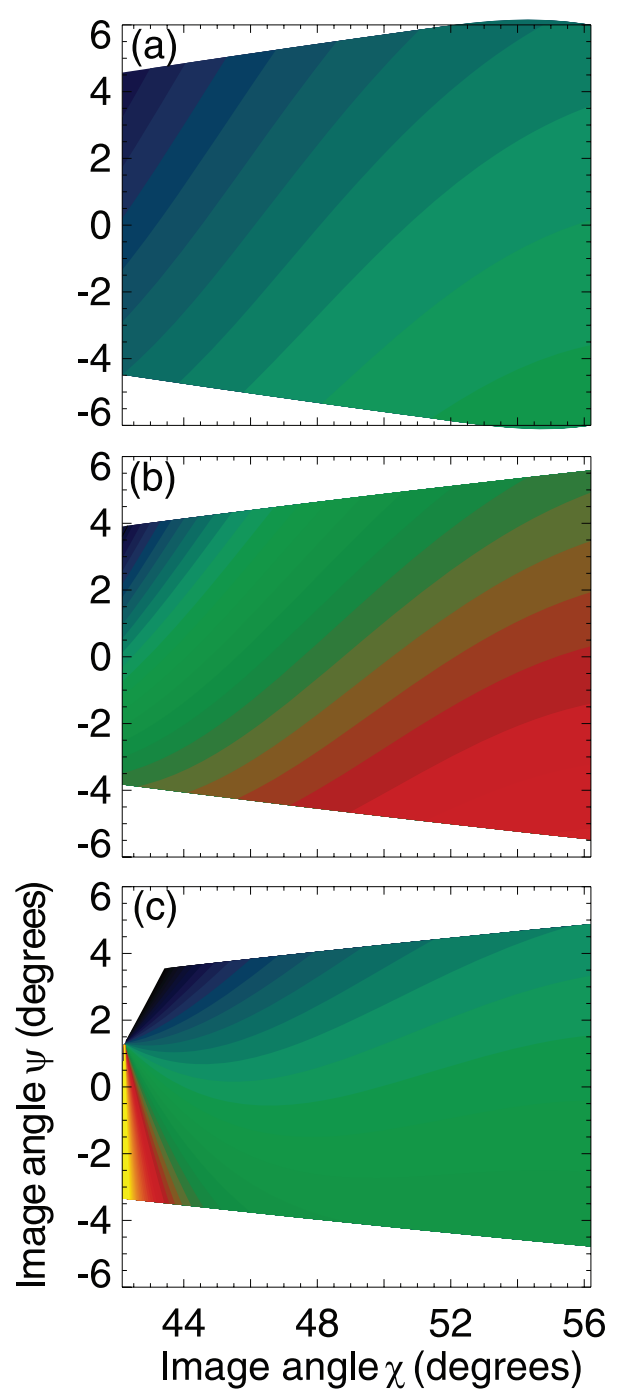

Fig. 10 (a)-(c): Calculated pitch angle distributions in vertical planes along lines a-c respectively as shown in Fig. 3 .

paign indicated some coating that could have contaminated the final polarization image. Thus it is clear that additional experiments and crosschecks are required to confirm these first results.

\section{Discussion and Future Work}

Polarization interferometers have a number of advantages for spectro-polarimetric imaging, including high throughput, simple and compact optics with easy alignment and 2-d imaging capability. By obviating the need for narrowband filters, the spectro-polarimeter described here allows MSE imaging for the first time. Importantly, the technique is insensitive to unpolarized or wideband polarized background contamination. There would also seem to be some sensitivity advantage in that the instrument is sensitive to 4 times the polarization inclination (see Eqs. (4) and (5)).

The preliminary results reported here suggest a number of cross-checks and validation experiments, including beam into gas calibration, reversal of field direction etc. which will be undertaken during dedicated experiments in future. Clearly it will be necessary to attempt to quantify and possibly unfold line-of-sight integration effects. In addition, a custom compound optical system to undertake double-spatial-heterodyne snapshot imaging will be implemented. Ultimately, a snapshot imaging system will be deployed for high speed imaging using a gated intensified camera for studies of MHD relaxation and other transient phenomena.

\section{Acknowledgements}

This work is supported by International Science Linkages established under the Australian Government's innovation statement, "Backing Australia's Ability". We also wish to thank Dr R. König for providing the imaging fibre array for these experiments.

[1] F.M. Levinton et al., Phys. Rev. Lett. 63, 2060 (1989).

[2] D. Wroblewski et al., Rev. Sci. Instrum. 61, 3552 (1990).

[3] B.W. Rice, Fusion Eng. Des. 34-35, 135 (1999).

[4] J. Howard, Plasma Phys. Control. Fusion 50, 125003 (18pp) (2008).

[5] M. Francon and S. Mallick, Polarization Interferometers (Wiley-Interscience, London, 1971). 